\title{
Role of the Hypothalamic Paraventricular Nucleus in Neuroendocrine Responses to Daylength in the Golden Hamster*
}

\author{
MICHAEL N. LEHMAN**, ERIC L. BITTMAN ${ }^{* * *}$ and SARAH WINANS NEWMAN \\ Reproductive Endocrinology Program and Department of Anatomy and Cell Biology, The University of Michigan, \\ Ann Arbor, MI 48109 (U.S.A.)
}

(Accepted January 24th, 1984)

Key words: hypothalamus - paraventricular nucleus - neuroendocrine relations

\begin{abstract}
Daylength regulates reproduction in golden hamsters through a mechanism which involves the pineal indoleamine, melatonin. Retinal input to the suprachiasmatic nucleus of the hypothalamus ( $\mathrm{SCN}$ ) and sympathetic innervation of the pineal are critical to the inhibition of reproduction by short photoperiods. Since the hypothalamic paraventricular nucleus (PVN) receives extensive input from the $\mathrm{SCN}$ in the rat, and may influence autonomic function via its brainstem and spinal cord projections, we studied the role of this nucleus in photoperiodically induced gonadal regression in the hamster. Bilateral electrolytic destruction of either the paraventricular nucleus (PVN) or suprachiasmatic nucleus (SCN) of the hypothalamus completely blocked testicular regression induced by either blinding or exposure to short days (10L:14D). Lesions in the retrochiasmatic hypothalamus (RCA) which may have interrupted the pathway of previously identified efferents from the SCN to the PVN were also effective in preventing short day-induced gonadal regression. Pineal melatonin content was measured in intact and lesioned hamsters sacrificed 3-5 h before lights on, at the time of the expected nocturnal peak. While SCN and RCA lesions significantly reduced pineal melatonin content, PVN lesions were still more effective in this regard. We conclude that the hamster's neuroendocrine response to photoperiod is mediated by neural pathways which include retinohypothalamic input to the SCN and efferents from this nucleus to the PVN which travel dorsocaudally through the retrochiasmatic area of the hypothalamus. Effectiveness of lesions restricted to the PVN suggests that direct projections from the PVN to spinal autonomic centers convey photoperiodic information which regulates pineal, and hence gonadal, function.
\end{abstract}

\section{INTRODUCTION}

Neuroendocrine function is influenced by daylength in a variety of seasonally breeding species ${ }^{15}$. For example, exposure of golden hamsters to photoperiods of less than $12.5 \mathrm{~h}$ light per day precipitates gonadal regression within 3 months ${ }^{14}$. This response is controlled by the pineal gland, which in turn is driven by its sympathetic innervation: removal of the pineal or the superior cervical ganglia eliminates the ability of short days to arrest reproductive function ${ }^{24}$. These procedures also eliminate the nocturnal rise in the synthesis and secretion of melatonin in several species ${ }^{6} \cdot 16,20.21 .25$. Since exogenous melatonin can replicate the reproductive effects of daylength in pinealectomized seasonal breeders, pineal secretion of this indole is believed to mediate photoperiodic control of gonadal function ${ }^{4}$.

Light influences pineal function in the rat via a retinohypothalamic projection to the suprachiasmatic nucleus of the hypothalamus $(\mathrm{SCN})^{1+.21}$. The efferent projections of this nucleus have been traced autoradiographically $3,18.30,34$ and consist of two major pathways: a ventral projection which courses caudally

\footnotetext{
* Portions of the work described in this manuscript were presented at the Thirteenth Annual Meeting of the Society for Neuroscience, November, 1983, Boston, MA, Abstract 96.6

** Present address and address for correspondence: Department of Anatomy, College of Physicians and Surgeons. Columbia University, New York, NY 10032, U.S.A. *** Present address: Division of Neuroendocrinology, Box 139. Rockefeller University, 1230 York Avenue, New York, NY 10021.
U.S.A. Address for reprint requests: S. W. Newman, Department of Anatomy and Cell Biology, The University of Michigan, Ann Ar-
bor. MI 48109. U.S.A.
} 
through the tuberal hypothalamus, the premammillary region, and the midbrain, and a dorsal projection through which fibers reach the paraventricular nuclei of the hypothalamus (PVN) and the thalamus, the dorsomedial hypothalamus (DMH), and the central gray $3,18,29,30,32,34,40$. Recent evidence suggests the dorsal pathway to the PVN may be critical to photic control of pineal function. Klein et al. ${ }^{17}$ reported that bilateral lesions centered on the PVN reduce nighttime pineal melatonin synthesis and nocturnal excretion of 6-hydroxymelatonin in rats. This is consistent with the neuroanatomical demonstration of direct projections from the PVN to the intermediolateral column of the rat thoracic spinal cord ${ }^{1,35}$, from which fibers which control sympathetic functions including pineal indole synthesis originate ${ }^{23}$.

Although data obtained in the rat suggest that the PVN-spinal connections control pineal indole metabolism, the function of the PVN has not been examined in a seasonal breeder in which photoperiod regulates reproduction through the pineal gland. In one such species, the golden hamster, lesions of the SCN and the surrounding periventricular hypothalamus eliminate reproductive responses to photoperiod $12,26,31$. Furthermore, initial studies of projections from the PVN to the spinal cord of the hamster suggest the existence of a pathway similar to that of the rat $^{9}$. The present experiments compared the effects of destruction of the hamster SCN and PVN on pineal melatonin content and testicular function in short photoperiods. The results suggest that retinohypothalamic input important to reproduction is relayed to the pineal via the PVN.

\section{METHODS}

Golden hamsters (Mesocricetus auratus) of the LVG/LAK strain were obtained from Lakeview laboratories for use in these studies. Animals were group caged in long photoperiods (14L:10D, lights on $20.00 \mathrm{~h}$ ) prior to experimental use. During the light phase, fluorescent bulbs generated an intensity of 1400 lux at the level of the cage floor. Bilateral electrolytic lesions were produced under pentobarbital anesthesia using a Grass LM-5 DC lesionmaker. Stainless-steel 00 insect pin electrodes were insulated except at the tip $(0.25 \mathrm{~mm})$ with epoxylite. PVN lesion coordinates were $7.3 \mathrm{~mm}$ below the dura, 0.25 $\mathrm{mm}$ posterior to bregma, $0.3 \mathrm{~mm}$ lateral to the midsagittal sinus. SCN lesions were placed $0.5 \mathrm{~mm}$ anterior to bregma, $0.1 \mathrm{~mm}$ lateral to the midline, and $7.9 \mathrm{~mm}$ below the dura. In experimental animals, $0.4 \mathrm{~mA}$ were passed for $15 \mathrm{~s}$; in sham-lesioned hamsters, no current was passed. Since no differences were observed in the gonadal or pineal responses of sham-lesioned and unoperated control hamsters, results from these groups were combined in statistical comparisons.

Photoperiodic treatments began approximately 3 weeks after the placement of lesions. In experiment 1 , individually caged hamsters were blinded by bilateral orbital enucleation under metofane anesthesia. Eleven weeks later, all hamsters were laparotomized under Nembutal anesthesia prior to transcardial perfusion with saline and formalin. Gonadal condition was determined by measurement of the maximum length and width of the left testis; the product of these numbers was divided by total body weight to provide a testis index (T.I.). This index has been shown in previous experiments 5.26 and confirmed in the present study to reflect accurately the spermatogenic and steroidogenic capacity of the testis.

In experiment 2 , hamsters were caged in pairs and transferred to a light-tight box in which a short photoperiod (10L:14D, lights on $22.00 \mathrm{~h}$ ) prevailed. Eleven weeks later, animals were rapidly decapitated under dim red illumination between 17.00 and $19.00 \mathrm{~h}$ (3-5 h prior to light onset on 10L:14D). The pineal gland was removed within 3 min of death and frozen in dry ice. Brains were removed and formalin-fixed without perfusion; frozen sections ( $60 \mu \mathrm{m}$ thick) were stained with cresyl violet for analysis of lesion placement. Pineal glands were subsequently sonicated in phosphate-buffered saline $(0.01 \mathrm{M}, \mathrm{pH} 7.0)$ prior to melatonin extraction and radioimmunoassay by the method of Tamarkin et al. ${ }^{39}$, except that chloroform extracts were washed with carbonate buffer $(\mathrm{pH}$ 10.25) and distilled water to remove indoles which might cross-react with the melatonin antibody. Antibody was obtained from Dr. M. D. Rollag. All samples were measured in a single radioimmunoassay, and values were corrected for extraction losses as estimated from quantitative recovery of a known amount of authentic melatonin $(82.5 \%)$. Testes were removed, trimmed of adhering fat, and weighed before fixation in Bouin's solution for histological ex- 
amination. Differences between groups were statistically evaluated by paired $t$-test.

\section{RESULTS}

Effects of SCN and PVN lesions on testicular function.

When inspected 11 weeks after blinding, the gonads of 4 control hamsters in experiment 1 were found to be uniformly regressed (T.I. $=0.63 \pm 0.04$, mean \pm S.E.M.). In contrast, 6 hamsters in which the PVN had been bilaterally destroyed prior to blinding had large testes $($ T.I. $=2.33 \pm 0.10 ; P<0.001$ vs sham-lesioned blinded controls). The testes of the PVN-lesioned hamsters were similar in size to those of sighted animals maintained in long days in our laboratory and in previous studies ${ }^{5.14 .26}$. Microscopic examination confirmed that the testes of blinded control hamsters were spermatogenically inactive and that the diameter of the seminiferous tubules was markedly reduced relative to PVN-lesioned animals.

Eleven weeks of exposure to 10L:14D resulted in dramatic testicular regression in sham-lesioned hamsters in experiment 2 (Fig. 1). Hamsters bearing bilateral lesions of the PVN or the SCN area had testes as heavy as those of animals maintained in long days $(P<0.001$ vs short-day controls; Fig. 1). Histological analysis confirmed corresponding differences of tubular diameter and spermatogenic activity between hamsters of the lesioned and control groups.

\section{Effects of SCN and PVN lesions on body weight}

Hamsters exposed to 10L:14D following bilateral destruction of the caudal PVN showed a substantial increase in body weight (Table I). This effect was not observed in blinded PVN-lesioned hamsters, in SCNlesioned hamsters maintained in 10L:14D. or in sham-lesioned and unoperated controls. As a consequence of the effect of PVN lesions on body weight, relative testis weights of $\mathrm{SCN}$-lesioned hamsters were significantly greater than those of PVN-lesioned animals at the end of 11 weeks of exposure to short days $(P<0.02)$. Absolute testis weights did not differ between the two groups $(P>0.3)$.

\section{Effects of SCN and PVN lesions on pineal melatonin content}

Pineal melatonin content differed markedly be-

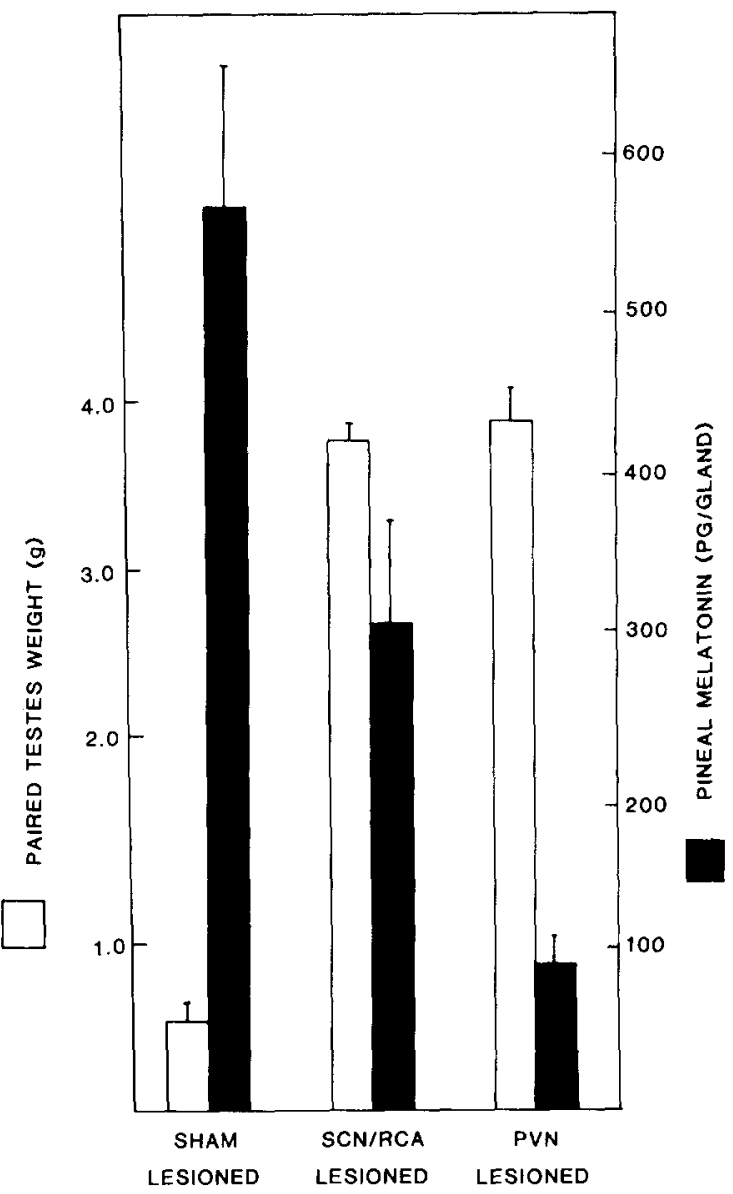

Fig. 1. Effects of lesions of the PVN $(n=7)$ and the SCN/retrochiasmatic periventricular hypothalamus $(\mathrm{SCN} / \mathrm{RCA}, \mathrm{n}=8$ ) on absolute testis weights $(g \pm S$.E.M. $)$ and pineal melatonin content (pg/gland \pm S.E.M.) in hamsters. Data were collected 11 weeks after lesion and transfer to short days. Hamsters were killed $3-5 \mathrm{~h}$ prior to lights off.

\section{TABLE I}

Effects of PVN and SCN lesions on body weight in male hamsters

Hamsters survived 11-14 weeks after lesion. Values reflect difference between preoperative and final body weights after 11 weeks of the specified photoperiodic treatments; numbers with different superscripts (a, b) differ significantly $(P<0.001)$.

\begin{tabular}{lcc} 
Group & $n$ & $\begin{array}{c}\text { Change in body } \\
\text { weight }(g)\end{array}$ \\
\hline Sighted hamsters in 10L:14D & & \\
$\quad$ Sham-lesioned & 4 & $+10.3 \pm 3.8^{\mathrm{a}}$ \\
$\quad$ SCN/RCA - lesioned & 8 & $+17.0 \pm 4.2^{\mathrm{a}}$ \\
PVN-lesioned & 7 & $+51.4 \pm 11.9^{\mathrm{h}}$ \\
Blinded hamsters & & \\
$\quad$ Sham-lesioned & & \\
PVN-lesioned & 4 & $+3.0 \pm 5.5^{\mathrm{a}}$ \\
& 6 & $+7.6 \pm 1.3^{\circ}$
\end{tabular}


tween groups in experiment 2 (Fig. 1). Whereas sham-lesioned animals generally exhibited the expected high levels of pineal melatonin 3-5 h prior to lights on, the pineals of PVN- and SCN-lesioned hamsters contained significantly lower levels of melatonin (both $P<0.005$ relative to controls; Fig. 1). Pineal melatonin content was significantly lower in PVN-lesioned than in SCN-lesioned hamsters killed at the same time of night $(P<0.005)$.

\section{Analysis of lesion placement}

With one exception, PVN lesions were bilaterally symmetrical and restricted primarily to this nucleus (Fig. 2A, B). In all cases the lateral magnocellular wing of the PVN was intact (see Fig. 2B, d), and in some animals the rostral one-third of the PVN was also largely spared (Fig. 2B, b). These lesions typically centered on the dorsomedial portion of the caudal two-thirds of the PVN with minimal damage to the adjacent $\mathrm{DMH}$ and midline thalamic nuclei (Fig. 2B). Significantly, none of these lesions impinged upon the $\mathrm{SCN}$. In the one exceptional blinded animal, the bilateral lesions were situated anteroventral to the main portion of the PVN and spared most
A

A. Normal Control

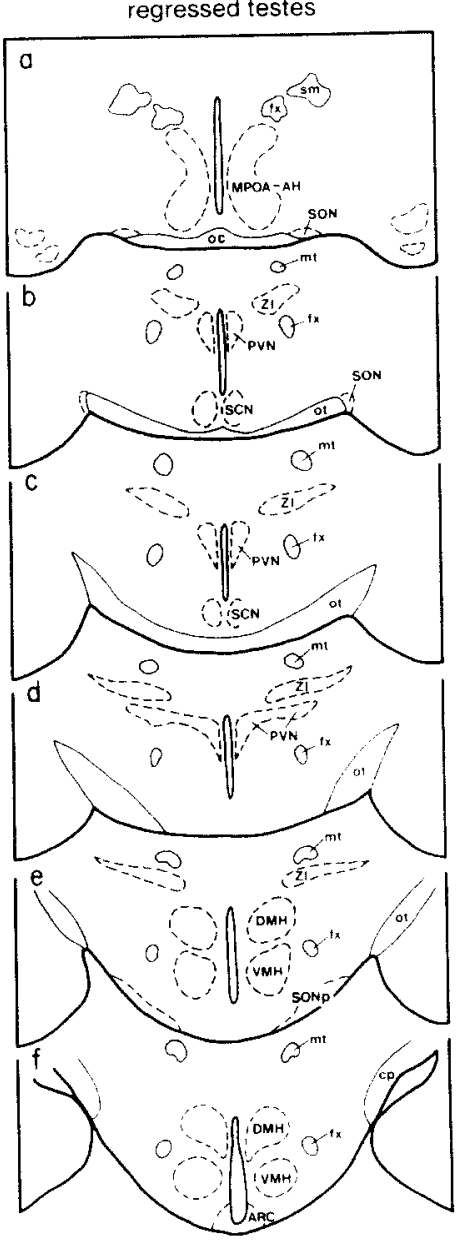

577
B. PVN Lesion

large testes

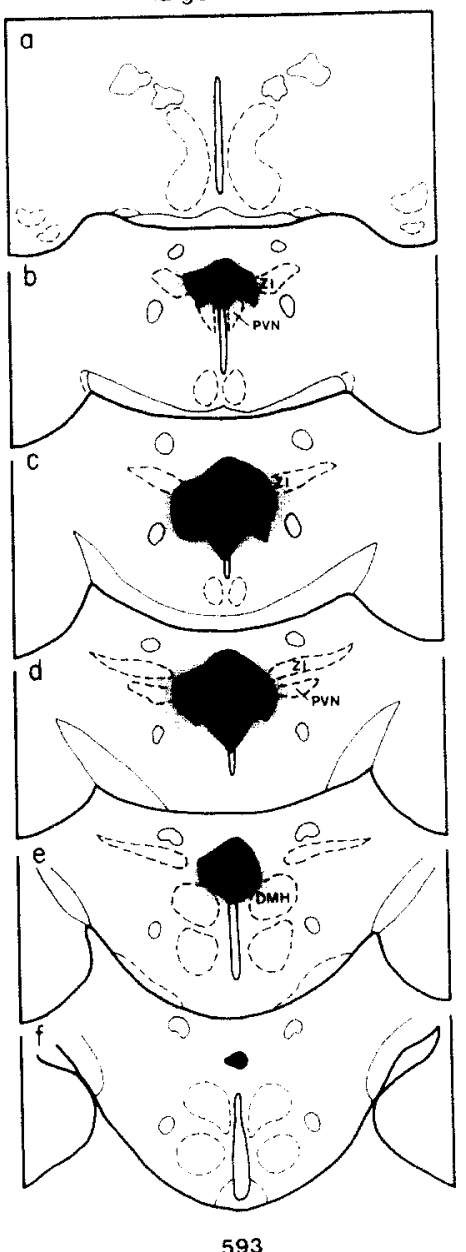

C. Misplaced PVN Lesion

partially regressed testes

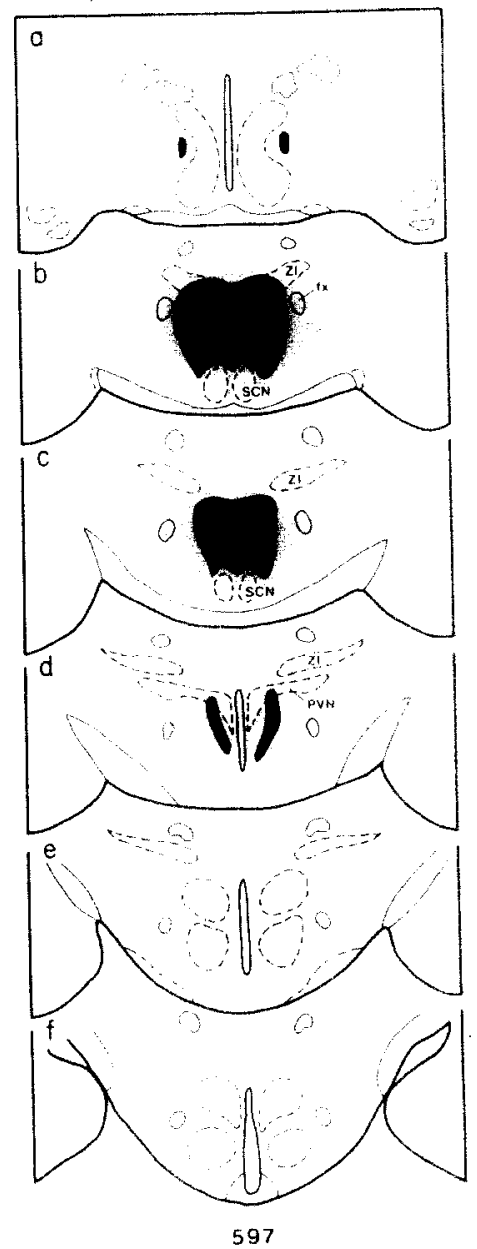

Fig. 2. Histological records of male hamsters with (A) sham lesions (animal number 577), (B) PVN lesions (animal number 593 , representative of 7 experimental animals) and (C) misplaced PVN lesion (animal number 597). The size and location of each lesion (necrotic and gliotic tissue) is shown in black on standard coronal sections through the hypothalamus from rostral to caudal (a-f, top to bottom). Testicular condition at end of experiment, 11 weeks after blinding or transfer to short days, is indicated at top. Abbreviations: ARC, arcuate nucleus; DMH, dorsomedial hypothalamic nucleus; $c p$, cerebral peduncle; $f x$, fornix; $M P O A-A H$, medial preoptic-anterior hypothalamic area; $\mathrm{mt}$, mammilothalamic tract; oc, optic chiasm; ot, optic tract; PVN, paraventricular nucleus; SCN, suprachiasmatic nucleus; sm, stria medullaris; SON, supraoptic nucleus; SONp, supraoptic nucleus, posterior division; VMH, ventromedial hypothalamic nucleus; ZI, zona incerta. 
A. SCN Lesion

large testes
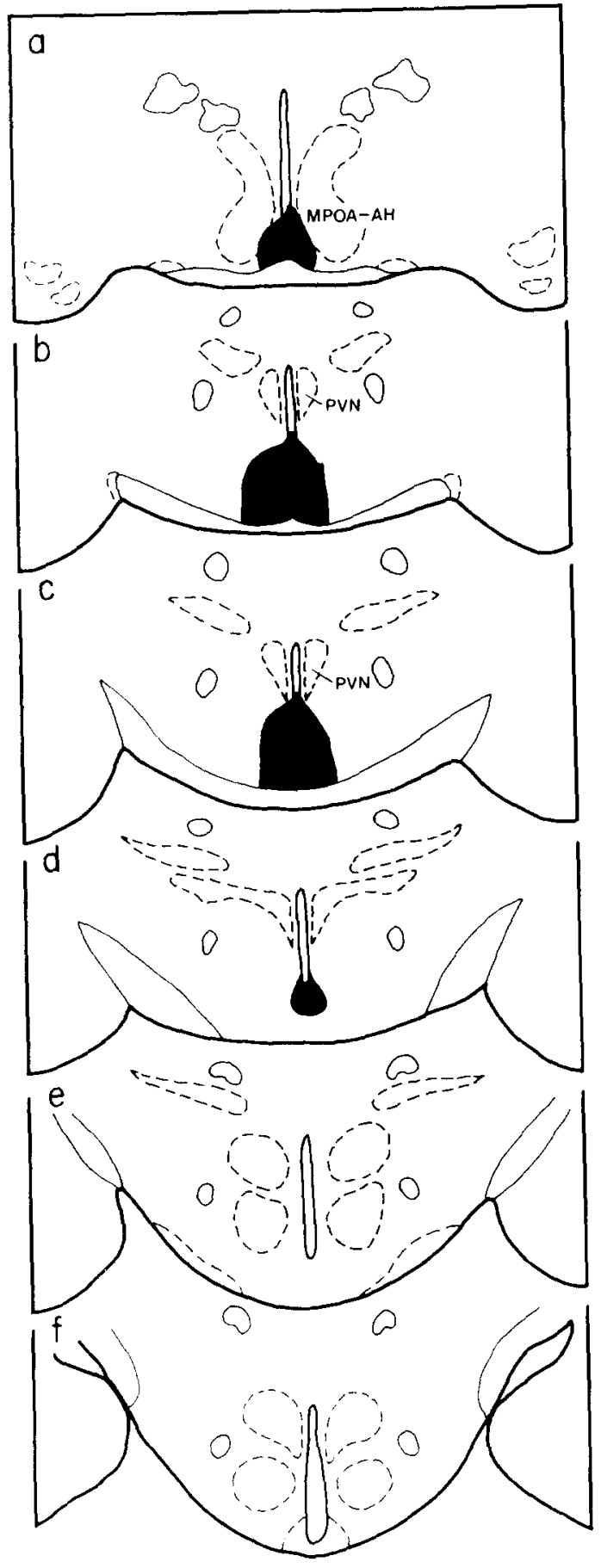

53
B. RCA Lesion

large testes
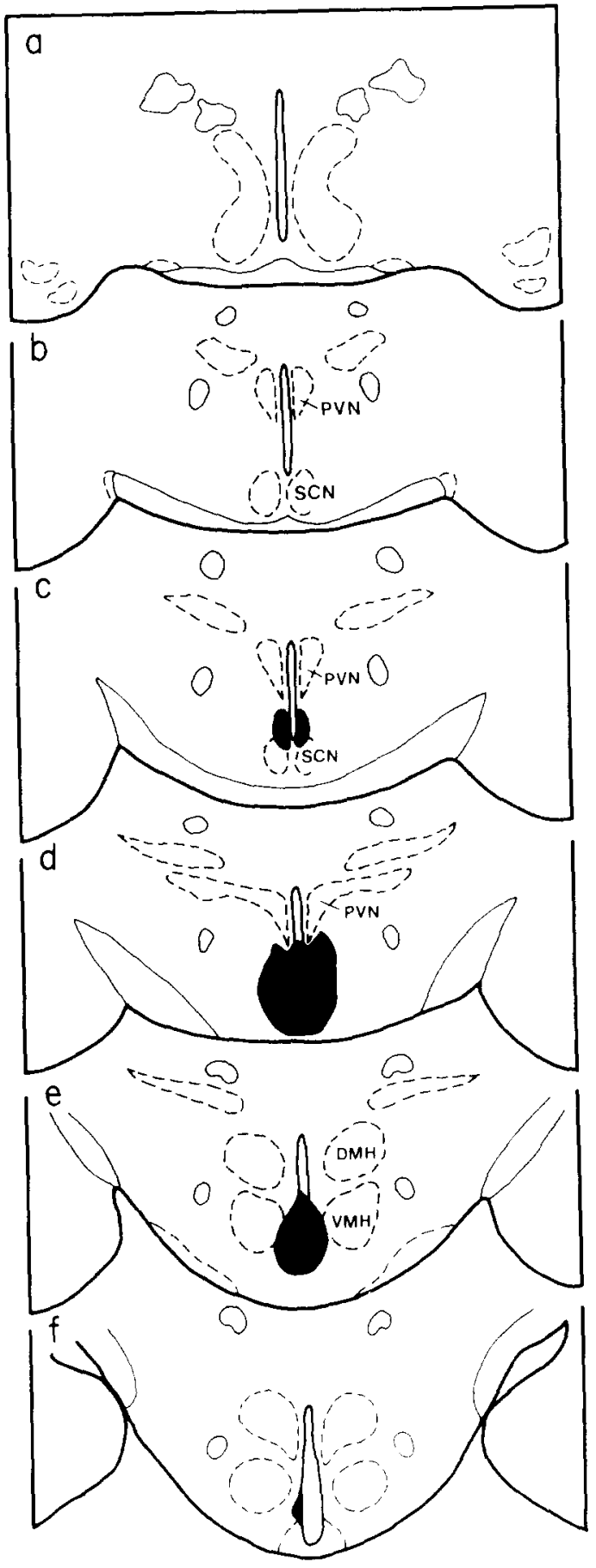

54

Fig. 3. Histological records of representative male hamsters with: (A) bilateral SCN lesions (animal number 53 , representative of 4 individuals); and (B) bilateral lesions in the retrochiasmatic area (RCA) (animal number 54, representative of 4 individuals). Format and abbreviations as in Fig. 2. 
of the mid- and caudal PVN (Fig. 2C). This individual exhibited substantial but incomplete testicular regression. It is noteworthy that in this animal, the lesions also bilaterally spared the periventricular zone immediately ventral to the caudal PVN (see Fig. 2C. d).

Lesions aimed at the SCN proved somewhat more variable in their distribution. In 4 of 8 animals, bilateral lesions completely destroyed the SCN (Fig. 3A). In the remaining hamsters, however, the lesions were centered more caudally in the retrochiasmatic area (RCA) and spared a majority of the SCN bilaterally (Fig. 3B). In these animals, bilateral destruction of tissue in this periventricular retrochiasmatic zone, situated immediately ventral to the caudal portion of the PVN, was adequate to block testicular regression and reduce nocturnal concentrations of pineal melatonin. Since SCN- and RCA-lesioned hamsters had testicular weights $(3.98 \pm 0.10$ and $3.75 \pm$ $0.12 \mathrm{~g}$, respectively) and pineal melatonin levels (378.6 \pm 94.6 and $234.9 \pm 84.8 \mathrm{ng} /$ gland, respectively) which did not differ significantly, data from these groups have been combined in Fig. 1

\section{DISCUSSION}

Bilateral lesions restricted to the PVN consistently blocked the effects of blinding and short day exposure on testicular function. The effects of PVN lesions resemble those of destruction of the $\mathrm{SCN}$ in this respect, as well as in their reduction of nocturnal pineal melatonin content at the time of peak expected values ${ }^{39}$. These findings are consistent with the proposal that identified $\mathrm{SCN}$ projections to the PVN control autonomic centers which regulate pineal function ${ }^{17}$. This study thus provides one of the clearest indications to date of a role for PVN-spinal connections in photoperiodic control of reproduction and supports the importance of melatonin in seasonal breeding cycles.

Dramatic elevations of body weight in PVN-lesioned hamsters may indicate a general disruption of autonomic function 19,28. Such weight gain was absent in SCN-lesioned hamsters maintained in 10L:14D and in blinded PVN-lesioned hamsters, suggesting a specific interaction between the PVN and photoperiod which is not secondary to changes in testicular condition.
The finding that the locus of effective PVN lesions lies at the middle and caudal portions of this nucleus is of particular interest. In the hamster and rat, the caudal parvocellular subdivisions of the PVN contain the densest concentration of cells projecting to the spinal cord $4,35,37,38$. In the rat, oxytocinergic input from the PVN is concentrated at spinal levels T1-T3. which provide the greatest input to the superior cervical ganglion 23,37 . Preliminary immunohistochemical studies of neurophysin localization in the PVN and spinal cord suggest a similar neurochemical organization may exist in the hamster (Lehman et al, unpublished data). An alternative explanation for the effectiveness of PVN lesions in the present study is that they interrupted SCN efferents which travel through the periventricular PVN to contact cells located caudally, e.g. in midbrain central gray or dorsal vagal complex, which in turn project to the cord. Since SCN efferents may also reach the central gray by way of fibers which course ventrolaterally, $\mathrm{PVN}$ and periventricular lesions which blocked testicular regression most likely spared some $\mathrm{SCN}$-central gray projections $3,18,311,32,34$.

Fortuitous misplaced lesions in a few animals (Figs, 2C and $3 \mathrm{~B}$ ) provide clues to the trajectory of the functional pathway linking the SCN to the PVN. This evidence suggests that critical fibers may course caudally from the SCN before turning dorsally in the periventricular zone of the retrochiasmatic area to reach the PVN. Such a dorsal pathway to the PVN is consistent with the results of autoradiographic tract tracing studies of SCN projections in the rat 3.18 .30 .34 and preliminary findings in the hamster of VIP-positive fibers coursing from cells of origin in the SCN through the retrochiasmatic area (Lehman, unpublished data). The effectiveness of lesions which spare the anterior and lateral portions of the PVN provides further information on the functionally relevant portions of this circuit.

Our findings are consistent with previous work in the rat indicating that the $\mathrm{SCN}$. the retrochiasmatic area, and the medial forebrain bundle participate in the generation of nocturnal rises of pineal melatonin synthesis ${ }^{16,21}$. Knife cuts placed caudal to the SCN would be expected to interrupt projections to the PVN, and lesions of the MFB are likely to eliminate axons descending from the PVN to the spinal cord ${ }^{33}$ While SCN lesions significantly reduced hamster pi- 
neal melatonin content at the time of peak expected values in the present study, they were significantly less effective than PVN lesions in this regard (Fig. 1). It is possible that destruction of the SCN or its efferents eliminates rhythmic influences on pineal function while permitting basal melatonin synthesis to continue: PVN lesions may more closely resemble superior cervical ganglionectomy in their complete elimination of activity in this sympathetic pathway. Alternatively, central lesions may have differentially altered the phase of rhythms in pineal melatonin content. Characterization of pineal melatonin content at intervals throughout the night in intact, PVN-lesioned, and SCN-lesioned hamsters would resolve these questions. Despite the differences in absolute levels of pineal melatonin during the latter part of the night, lesions of the PVN and the SCN region were equally effective in blocking gonadal regression. This is consistent with evidence that the rhythmicity, rather than the total amount, of melatonin secretion may control reproductive responses to photoperiod $4,7,8$.

The blockade of gonadal responses to daylength by lesions of the PVN suggests that other areas which may receive SCN input and project to the spinal cord. such as the lateral hypothalamus ${ }^{3,9,18,310,34}$ and the retrochiasmatic region 27.36 , are not adequate to maintain the pineal-mediate photoperiodic response. It remains possible, however, that efferents of the SCN other than those which project to the PVN may participate in the discrimination of daylength through non-autonomic systems. It is also possible that the PVN plays an additional role in the response as a target of secreted melatonin. While these hypotheses cannot be ruled out and should be tested directly5.

\section{REFERENCES}

1 Armstrong, W. E., Warach, S., Hatton, G. I. and McNeill, T. H. Subnuclei in the rat hypothalamic paraventricular nucleus: a cytoarchitectural, horseradish peroxidase, and immunocytochemical analysis. Neuroscience, 5 (1980) $1931-1958$.

2 Aschoff, J. A. and Wever. R.. Human circadian rhythms: a multioscillatory system. Fed. Proc., 35 (1976) 2326-2332.

3 Berk, M. I. and Finkelstein. J. A., An autoradiographic determination of the efferent projections of the suprachiasmatic nucleus of the hypothalamus, Brain Research, 226 (1981) $1-13$

4 Bittman. E. L. Melatonin and photoperiodic time meas- none of the evidence now available supports them.

Hamsters utilize endogenous circadian rhythms to discriminate daylength ${ }^{10}$. Experimental manipulation of the phase relationship between endogenous oscillations and the light-dark cycle which entrains them can result in reproductive responses inappropriate to the photoperiod ${ }^{11.13}$. It is possible, therefore, that the absence of gonadal responses to short days in PVN-lesioned hamsters is attributable to alterations in entrainment. The finding in experiment 1 that PVN lesions blocked testicular regression in blinded hamsters, however, suggests that the protection of gonadal function is not secondary to alteration of the phase angle of entrainment to short light-dark cycles. This conclusion is supported by the recent observations of Pickard and Turek22 that similar lesions did not affect circadian rhythms of locomotor activity in hamsters. The role and functional organization of different SCN efferents in photoperiodism and circadian rhythmicity require further study. A detailed examination of the role of the PVN in skeletal and autonomic rhythms may reveal fundamental properties of the neural basis of circadian organization.

\section{ACKNOWLEDGEMENTS}

We are grateful to Ms. Mary Nelson for her assistance in histology and animal care, and to Dr. Steven M. Yellon for his help in removing pineal glands. This research was supported by N.I.H. Grants HD11311, HD05914 and HD07048. E.L.B. was also supported by the Michigan Society of Fellows.

urement: evidence from rodents and ruminants. In R, J Reiter (Ed.), The Pineal Gland, Raven Press, NY, 1984. pp. $155-192$.

5 Bittman, E. L., Goldman, B. D. and Zucker, I., Testicular responses to melatonin are altered by lesions of the suprachiasmatic nuclei in golden hamsters. Biol. Reprod., 21 (1979) 647-656.

6 Bittman, E. L., Hopkins. J. and Karsch, F. 1. Role of the pineal gland in ovine photoperiodism: regulation of scasonal breeding and negative feedback effects of estradiol upon LH secretion. Endocrinology, 113 (1983) 329-336.

7 Bittman, E. L. and Karsch, F. J., Nightly duration of pineal melatonin secretion determines the response to daylength in the ewe. Biol. Reprod. 30(1984) 585-549.3. 
8 Carter, D. S. and Goldman, B. D., Antigonadal effects of timed melatonin infusion in pinealectomized male Djungarian hamsters (Phodopus sungorus sungorus): duration is the critical parameter. Endocrinology, 113 (1983) 1261-1267.

9 Don Carlos, L. L. and Finkelstein, J. A., Hypothalamic projections to the spinal cord in the hamster, Neurosci. Abstr., 8 (1982) 218 (Abstract 58.17).

10 Elliott, J. A., Circadian rhythms and photoperiodic time measurement, Fed. Proc., 35 (1976) 2339-2346.

11 Elliott, J. A., Circadian rhythms, entrainment, and photoperiodism in the syrian hamster. In B. K. Follett and D. E. Follett (Eds.), Biological Clocks in Seasonal Reproductive Cycles, Wright, Bristol, UK, 1981, pp. 203-217.

12 Eskes, G. A., Wilkinson, M. and Rusak, B., Neural control of hamster seasonal breeding cycles, Biol. Reprod., 26, Suppl. 1 (1982) 97A (Abstract 122).

13 Eskes, G. A. and Zucker, I., Photoperiodic regulation of the hamster testis: dependence on circadian rhythms, Proc. nat. Acad. Sci. U.S.A., 75 (1978) 1034-1038.

14 Gaston, S. and Menaker, M., Photoperiodic control of hamster testis, Science, 158 (1967) 925-928.

15 Hoffmann, K., Photoperiodism in vertebrates. In J. A. Aschoff (Ed.), Handbook of Behavioral Neurobiology, Vol. 4. Plenum, NY, 1981, pp. 449-473.

16 Klein, D. C. and Moore, R. Y., N-acetyltransferase and 5 hydroxyindole-O-methyltransferase: control by the retinohypothalamic tract and the suprachiasmatic nucleus, Brain Research, 174 (1979) 245-262.

17 Klein, D. C., Smoot, R., Weller, J. L., Higa, S., Markey, S. P., Creed, G. J. and Jacobowitz, D. M., Lesions of the paraventricular nucleus area of the hypothalamus disrupt the suprachiasmatic-spinal cord circuit in the melatonin rhythm generating system, Brain Res. Bull., 10 (1983) $647-651$.

18 Kucera, P. and Favrod, P., Suprachiasmatic nucleus projection to mesencephalic central grey in the woodmouse (Apodemus sylvaticus L.), Neuroscience, 4 (1979) 1705-1715.

19 Leibowitz, S. F., Hammer, N. J. and Chang, K., Hypothalamic lesions produce overeating and obesity in the rat. Physiol. Behav., 27 (1981) 1031-1040.

20 Lincoln, G. A. and Short, R. V., Seasonal breeding: nature's contraceptive, Rec. Prog. horm. Res., 36 (1980) $1-52$.

21 Moore, R. Y. and Klein, D. C., Visual pathways and the central neural control of circadian rhythms in pineal serotonin N-acetyltransferase activity, Brain Research, 71 (1974) $17-33$

22 Pickard, G. E. and Turek, F. W., Ablation of the hypothalamic paraventricular nucleus prevents short-day induced gonadal regression in the golden hamster without eliminating the circadian rhythm of locomotor activity, Neurosci. Abstr., 13:185.13.

23 Rando, T. A., Bowers, C. W. and Zigmond, R. E., Localization of neurons in the rat spinal cord which project to the superior cervical ganglion, J. comp. Neurol., 196 (1981) 73-83.

24 Reiter, R. J. and Hester, R. J., Interrelationships between the pineal gland, the superior cervical ganglion, and the photoperiod in the regulation of the endocrine systems of hamsters, Endocrinology, 79 (1966) 1168-1170.

25 Reppert, S. M. and Klein, D. C., Mammalian pineal gland: basic and clinical aspects. In M. Motta (Ed.), The Endocrine Functions of the Brain, Raven Press, NY, 1980, pp. 327-371.

26 Rusak, B and Morin, L. P., Testicular responses to photoperiod are blocked by lesions of the suprachiasmatic nucleus in the golden hamster, Biol. Reprod., 15 (1976) 366-374.

27 Saper, C. B., Loewy, L. W. and Cowan, W. M., Direct hypothalamo-hypophyseal connections, Brain Research, 117 (1976) 305-312.

28 Sawchenko, P. E., Gold, R. M. and Leibowitz, S. F., Evidence for vagal involvement in the eating elicited by adrenergic stimulation of the paraventricular nucleus. Brain Research, 225 (1981) 249-269.

29 Silverman, A. J., Hoffman, D. L. and Zimmerman, E. A., The descending afferent connections of the paraventricular nucleus of the hypothalamus (PVN), Brain Res. Bull. 6 (1981) 47-61.

30 Stephan, F. K., Berkley, D. J. and Moss, R. L., Efferent connections of the rat suprachiasmatic nucleus, Neuroscience, 6 (1981) 2625-2641.

31 Stetson, M. H. and Watson-Whitmyre, M., Nucleus suprachiasmaticus: the biological clock in the hamster? Science, 191 (1976) 197-199.

32 Sonofriew, M. V. and Weindl, A., Projections from the parvocellular vasopressin- and neurophysin-containing neurons of the suprachiasmatic nucleus, Amer. J. Anat. 153 (1978) 391-430.

33 Swanson, L. W., Immunocytochemical evidence for a neurophysin-containing autonomic pathway arising in the paraventricular nucleus of the hypothalamus, Brain Research, 128 (1977) 346-353.

34 Swanson, L. W. and Cowan, W. M., The efferent projections of the suprachiasmatic nucleus of the hypothalamus. J. comp. Neurol., 160 (1975) 1-12

35 Swanson, L. W. and Kuypers, H. G. J. M., The paraventricular nucleus of the hypothalamus: cytoarchitectonic subdivisions and organization of projections to the pituitary, dorsal vagal complex, and spinal cord as demonstrated by retrograde fluorescence double-labelling methods, J. comp. Neurol., 194 (1980) 555-570.

36 Swanson, L. W. and Kuypers, H. G.J. M., A direct projection from the ventromedial nucleus and retrochiasmatic area of the hypothalamus to the medulla and spinal cord of the rat, Neurosci. Lett., 17 (1980) 307--312.

37 Swanson. L. W. and McKellar, S., The distribution of oxytocin- and neurophysin-stained fibers in the spinal cord of the rat and monkey, J. comp. Neurol., 188 (1979) 87-106.

38 Swanson, L. W. and Sawchenko, P. E. Hypothalamic integration: organization of the paraventricular and supraoptic nuclei, Ann. Rev. Neurosci., 6(1983) 269-324.

39 Tamarkin, L., Reppert, S. M. and Klein, D. C., Regulation of pineal melatonin in the syrian hamster, Endocrinology. 104 (1979) 385-389.

40 Tribollet, E. and Dreifuss, J. J., Localization of neurones projecting to the hypothalamic paraventricular nucleus area of the rat: a horseradish peroxidase study, Neuroscience, 6 (1981) 1315-1328. 